\title{
Primary Tumor Levels of Human Tissue Kallikreins Affect Surgical Success and Survival in Ovarian Cancer Patients
}

\author{
Julia Dorn, ${ }^{1}$ Manfred Schmitt, ${ }^{1}$ Ronald Kates, ${ }^{1}$ Barbara Schmalfeldt, ${ }^{1}$ Marion Kiechle, ${ }^{1}$ \\ Andreas Scorilas, ${ }^{2}$ Eleftherios P. Diamandis, ${ }^{3,4,5}$ and Nadia Harbeck ${ }^{1}$
}

\begin{abstract}
Purpose: Proteolytic factors of the human tissue kallikrein (hK) family and the plasminogen activation system play a key role in tumor progression in various malignancies. We determined antigen levels of urokinase-type plasminogen activator (uPA), its inhibitor PAl-1, and hK5-8, hK10, hK11, and hK13 by ELISA in primary tumor tissue extracts of 142 International Federation of Gynecology and Obstetrics (FIGO) I to IV ovarian cancer patients (median follow-up 41 months). Results: After radical surgery, absence of macroscopically visible residual tumor (RT) was achieved in 72 patients; all patients received postoperative platinum-containing chemotherapy. Significant univariate predictors of poor progression-free survival (PFS) were RT (>0), FIGO stages (III/IV versus I/II/III), ascites volume $>500 \mathrm{~mL}$, nodal status, and the difference between PAI-1 and uPA (fractionally ranked). In multivariate analysis, significant independent factors for poor PFS were RT [hazard ratio (HR), 4.53] and low hK11 fractional rank (HR, 0.30). Univariate predictors of poor overall survival were RT, FIGO stages, nodal status, ascites volume, nuclear grade, and low hK10 and hK13. In multivariate analysis, significant independent factors for poor overall survival were RT (HR, 7.49), ascites (HR, 1.97), and low hK10 (HR, 0.196). We constructed a multivariate scoring model estimating $\mathrm{RT}$ probability, based on ascites [odds ratio (OR), 13.1], nuclear grade $(\mathrm{OR}, 2.92)$, hK6 (OR, 8.54), and hK13 (OR, 0.14), with good in-sample predictive performance (area under receiver operating characteristic, 0.833 ).

Conclusions: In view of risks and benefits of radical surgery, such a score could support preoperative risk stratification and identify candidates for alternative therapeutic strategies. These results highlight the distinct roles of the hKs for different disease end points in ovarian cancer and their potential to support individualized therapy decisions.
\end{abstract}

Epithelial ovarian cancer, with the highest mortality rate of all gynecologic malignancies, is seldom diagnosed before the tumor is disseminated throughout the entire abdominal cavity. State-of-the-art therapy consists of radical debulking surgery with the goal of complete tumor resection, followed by combination chemotherapy. However, therapeutic interventions are associated with considerable morbidity. In patients with advanced disease, radical surgery leads to $\sim 3 \%$ mortality,

\footnotetext{
Authors' Affiliations: 'Department of Obstetrics and Gynecology, Klinikum rechts der Isar, Technical University of Munich, Munich, Germany; ${ }^{2}$ NCS Research Demokritos, National University of Athens, Athens, Greece; ${ }^{3}$ Department of Pathology and Laboratory Medicine, Mount Sinai Hospital; ${ }^{4}$ Department of Clinical Biochemistry, University Health Network and Toronto Medical Laboratories; and ${ }^{5}$ Department of Laboratory Medicine and Pathobiology, University of Toronto, Toronto, Ontario, Canada

Received 10/13/06; revised 12/1/06; accepted 12/18/06

Grant support: Wilhelm Sander Foundation grant 2001.009.2 (N. Harbeck).

The costs of publication of this article were defrayed in part by the payment of page

charges. This article must therefore be hereby marked advertisement in accordance with 18 U.S.C. Section 1734 solely to indicate this fact.

Requests for reprints: Nadia Harbeck, Department of Obstetrics and Gynecology, Klinikum rechts der Isar, Technical University of Munich, Ismaninger Strasse 22 D-81675 Munich, Germany. Phone: 49-89-4140-6658; Fax: 49-89-4140-4846; E-mail: nadia.harbeck@Irz.tum.de.

(C) 2007 American Association for Cancer Research

doi:10.1158/1078-0432.CCR-06-2482
}

and two thirds eventually suffer recurrence and death anyway. Incorporating tumor-associated biomarkers into selection of candidates for novel primary clinical therapy approaches could improve survival or at least reduce needless morbidity. Conceivable options enabled by better primary scoring might include preoperative (neoadjuvant) chemotherapy or a second-effort surgical approach after incomplete primary surgery.

Numerous studies have focused on improved understanding of the underlying tumor biology in ovarian cancer. Tumor proteases, especially urokinase-type plasminogen activator (uPA), a serine protease, and its inhibitor PAI-1, may play a key role in invasion and metastasis, because both uPA and PAI1 antigen levels are elevated in ovarian cancer tissue compared with benign ovarian tumors $(1-3)$. Moreover, $\mathrm{UPA}$ and PAI-1 are strong prognostic markers in advanced ovarian cancer $(2,4)$, especially in patients without residual tumor (RT) mass.

Evidence for involvement of the tissue kallikrein serine protease family in ovarian cancer spread is emerging. The tissue kallikrein family genes are clustered (5) on chromosome 19q13.4. Thus far, 15 human tissue kallikrein (hK) protease proteins have been identified. In ovarian cancer tissue, hK4-8, hK10, hK11, hK13, and hK15 are differentially expressed at mRNA and protein levels (6). Some tissue kallikrein proteins (hK5, hK6, hK8, hK10, and hK11) seem to be useful serum diagnostic markers in ovarian cancer with complementary 
value to that of the tumor marker CA125 (7). Serum hK6 protein levels decrease in $68 \%$ of patients after surgery (8); hK10 is elevated in serum of ovarian cancer patients, but not in patients with benign gynecologic diseases. High hK10 in serum has been associated with late-stage, advanced-grade, suboptimal tumor debulking as well as shorter progression-free survival (PFS) and overall survival (OS; ref. 9). In ovarian cancer tissue extracts, hK5, hK6, hK7, and hK10 have been reported as markers of poor prognosis: hK5 is significantly elevated in ovarian cancer cytosols, compared with lowmalignant-potential tumors. Higher hK5 levels are associated with advanced disease and significantly shorter PFS and OS (10), whereas high levels of hK8, hK11, and hK13 are associated with less aggressive and less advanced disease $(5,11)$. hK11-positive tumors were associated with early stage and low tumor grade; high tumor levels of hK11 and hK13 imply longer PFS and OS $(12,13)$. Besides their prognostic effect, elevated hK10 and hK13 have been associated with optimal debulking $(9,13)$, and elevated hK11 has been associated with response to chemotherapy (14).

Despite this emerging evidence for their role in disease processes, there is no clear basis yet for incorporating these proteolytic factors into evidence-based decision criteria in ovarian cancer. Key steps are to assess their relative and combined effects on surgical success and survival in a homogeneous, representative patient cohort, to determine which factors are essential for improving the quality of existing models, and to construct multivariate models suitable for future validation and clinical decision support. This article evaluates the clinical effect of seven tissue kallikreins (hK5, hK6, hK7, hK8, hK10, hK11, and hK13) as well as that of uPA and PAI-1 in extracts of tumor tissue samples obtained from primary ovarian cancer patients; the results are translated into clinically applicable, multivariate scoring models for surgical success and survival, including both established clinical variables and proteolytic factors. The goal of combining all this information is to support better clinical decisions and ultimately to improve both survival and quality of life for patients.

\section{Materials and Methods}

Patients. Surgery was done on 142 patients (median age 57.5 years; 19-85 years) with early and advanced ovarian carcinoma [International Federation of Gynecology and Obstetrics (FIGO) I-IV] between 1985 and 1999 at the Department of Obstetrics and Gynecology, Technical University of Munich. The study was approved by the local ethics committee; all patients gave written informed consent. All patients received postoperative platinum-containing chemotherapy. Median follow-up in patients still alive at time of analysis was 41 months (1-125 months). The collective contains 41 patients (all FIGO IIIc) who were also included in an earlier report (2). Ascites volume was estimated preoperatively by ultrasound. Survival and RT statistics are included in Table 1.

Tissue extraction. Ovarian tumor tissue specimens were extracted as previously described (3); fresh tumor tissue samples were inspected by the pathologist immediately after surgery, snap-frozen, and stored in liquid nitrogen. For tissue disintegration, deep-frozen ovarian tissue was pulverized using the microdismembrator II (Braun-Melsungen, Melsungen, Germany); the still-frozen powder was then suspended in extraction buffer $\left(4^{\circ} \mathrm{C}\right)$ containing $0.02 \mathrm{~mol} / \mathrm{L}$ Tris-HCl $(\mathrm{pH} 8.5)$, $0.125 \mathrm{~mol} / \mathrm{L} \mathrm{NaCl}$, and $1 \%(\mathrm{w} / \mathrm{v})$ nonionic detergent Triton X-100 (15).
After gentle rotation for $15 \mathrm{~h}$ at $4^{\circ} \mathrm{C}$, followed by ultracentrifugation for $45 \mathrm{~min}$ at $100,000 \times g\left(4^{\circ} \mathrm{C}\right)$, the supernatant (extract) was collected, aliquoted ( $50 \mu \mathrm{L}$ portions), and stored in liquid nitrogen until further use.

Determination of antigen levels of uPA, PAI-1, and tissue kallikreins in ovarian cancer tissue extracts. Protein concentrations of the extracted tissue specimens were determined using the BCA Protein Assay reagent kit (Pierce, Rockford, IL). Antigen levels of the proteolytic factors uPA, its inhibitor PAI-1, and seven tissue kallikreins (hK5, hK6, hK7, hK8, hK10, hK11, hK13) were determined by ELISA in primary ovarian cancer tissue extracts. uPA and PAI- 1 antigen levels were measured using ELISA kit Imubind \#894 and \#821 (American Diagnostica, Inc., Stamford, CT), as described previously (16). Antigen concentrations of hK proteins were quantified by using highly sensitive and specific sandwich-type, in-house immunoassays (17). Capture antibodies were generated by immunizing mice with recombinant hK protein (monoclonal for hK5, hK11, and hK13; polyclonal for hK6, hK7, hK8, and hK10), detection antibodies by immunizing rabbits (all polyclonal). Lower and upper detection limits were 0.1 to $50 \mathrm{ng} / \mathrm{mL}$ for hK5 (18), 0.5 to $200 \mathrm{ng} / \mathrm{mL}$ for hK6 (19), 0.2 to $10 \mathrm{ng} / \mathrm{mL}$ for hK7 (20), 0.2 to $20 \mathrm{ng} / \mathrm{mL}$ for hK8 (21), 0.05 to $10 \mathrm{ng} / \mathrm{mL}$ for hK10 (22), 0.1 to $50 \mathrm{ng} / \mathrm{mL}$ for hK11 (12), and 0.05 to $20 \mathrm{ng} / \mathrm{mL}$ for hK13 (23). No cross-reactivity of any of the hK-ELISAs with other members of the hK family was detected. Analyte levels measured in the extracts were expressed in nanograms of analyte per milligram of protein.

Statistical methods. Outcome variables were PFS, OS, and RT presence, defined as one if macroscopic RT mass was visible and zero if completely absent. Ascites volume, age, nuclear grade, and nodal status were coded as binary variables (ascites $>500 \mathrm{~mL}$ versus less; age $>60$ years versus younger; nuclear grade, G3 versus G1/G2; nodal status 0 for $\mathrm{N}_{0}$, otherwise 1). FIGO status was coded by three binary indicators: II/III/IV versus I, III/IV versus I/II, and IV versus I/II/III. Table 1. Clinical and histomorphologic
characteristics of the cohort

\begin{tabular}{lc} 
& $\boldsymbol{n}(\mathbf{\%})$ \\
\hline FIGO stage & \\
I & $25(17.6)$ \\
II & $10(7.0)$ \\
III & $78(54.9)$ \\
IV & $29(20.5)$ \\
Nuclear grade & \\
G1 & $14(9.9)$ \\
G2 & $41(28.9)$ \\
G3 & $86(60.5)$ \\
Unknown & $1(0.7)$ \\
Relapsed & $73(51.4)$ \\
No & $69(48.6)$ \\
Yes & \\
Deceased & $78(54.9)$ \\
No & $64(45.1)$ \\
Yes & \\
RT mass (cm) & $72(50.7)$ \\
0 & $55(38.7)$ \\
$>0$ & $15(10.6)$ \\
Unknown & \\
Ascitic fluid volume & $36(25.4)$ \\
No ascites & $47(33.1)$ \\
$\leq 500$ mL & $48(33.8)$ \\
$>500$ mL & $11(7.7)$ \\
Unknown & $17(12.0)$ \\
Response to chemotherapy & $65(45.8)$ \\
Progression & $60(42.2)$ \\
No change & \\
Unknown & \\
\hline & \\
\hline
\end{tabular}


Table 2. Population distribution characteristics of antigen levels for UPA, PAI-1, and tissue kallikreins hK5, hK6, hK7, hK8, hK10, hK11, and hK13 in primary ovarian cancer measured in tissue extracts by ELISA

\begin{tabular}{|c|c|c|c|c|c|c|c|}
\hline \multirow{2}{*}{$\begin{array}{l}\text { Antigen } \\
\text { (ng/mg protein) }\end{array}$} & \multirow[t]{2}{*}{$n$} & \multirow{2}{*}{$\begin{array}{l}\text { Below detection } \\
\text { limit }\end{array}$} & \multirow[t]{2}{*}{ Range } & \multirow[t]{2}{*}{ Mean (SD) } & \multicolumn{3}{|c|}{ Percentile } \\
\hline & & & & & 25th & Median & 75th \\
\hline uPA & 141 & 0 & $0.02-24.0$ & $1.7(2.6)$ & 0.4 & 0.9 & 1.8 \\
\hline PAI-1 & 141 & 1 & $0.0-389.8$ & $32.8(54.7)$ & 6.0 & 14.7 & 34.4 \\
\hline hK5 & 142 & 58 & $0.0-219.6$ & $3.1(18.6)$ & 0.0 & 0.39 & 1.9 \\
\hline hK6 & 141 & 7 & $0.0-432.7$ & $20.5(44.2)$ & 1.8 & 9.4 & 19.9 \\
\hline hK7 & 142 & 31 & $0.0-283.6$ & $7.8(26.0)$ & 0.2 & 2.1 & 5.2 \\
\hline hK8 & 142 & 19 & $0.0-477.8$ & $28.9(58.7)$ & 1.4 & 9.5 & 24.7 \\
\hline hK10 & 141 & 21 & $0.0-233.6$ & $10.7(22.4)$ & 1.3 & 3.9 & 12.0 \\
\hline hK11 & 141 & 33 & $0.0-155.4$ & $8.3(21.4)$ & 0.1 & 1.5 & 6.2 \\
\hline hK13 & 141 & 88 & $0.0-18.4$ & $0.6(2.2)$ & 0.0 & 0.0 & 0.3 \\
\hline
\end{tabular}

NOTE: The number of measurements "below detection limit" is also given (not to be confused with "missing" values).

In view of their long-tailed distributions (Table 2), all antigen levels (uPA, PAI-1, and the tissue kallikreins) were coded as fractional population ranks. The fractional rank difference between PAI-1 and uPA was also coded and considered as a separate indicator. Mann-Whitney or Kruskal-Wallis tests were computed for associations between continuous and categorical variables. The effects of the different factors on PFS and OS were expressed as hazard ratios (HR) with respect to the above coding and were estimated by Cox proportional hazards regression using forward selection. Univariate and multivariate Cox models were estimated including clinical factors and fractionally ranked antigen levels, entered as continuous variables. Kaplan-Meier curves and log-rank statistics were also computed.

Effect of factors on RT was estimated by univariate/multivariate binary logistic regression and expressed as unadjusted/adjusted odds ratios (OR), respectively. For each patient, a score (estimated probability of RT) was obtained and used to construct a receiver operating characteristic (ROC; sensitivity / specificity). The area under ROC provides an in-sample measure of model quality. Analyses were done using SPSS; $P$ values $<0.05$ were reported as significant.

\section{Results}

The present investigation is a retrospective study of the cohort with respect to clinical and proteolytic factors. Table 1 summarizes clinical and histomorphologic characteristics.
Quantitative assessment of UPA, PAI-1, and tissue kallikreins. Table 2 summarizes the antigen distributions for uPA, PAI-1, and the seven tissue kallikreins (hK5, hK6, hK7, hK8, hK10, hK11, hK13). All are long-tailed, as illustrated by the mean, median, and selected percentile values. Table 2 also shows the number of patients for which each tissue kallikrein was not present above the limits of detection. In all of the tissue extracts examined, uPA antigen was detected; PAI-1 was detected in all but one sample.

There is no significant difference in any antigen levels between early (FIGO I/II) or advanced (FIGO III/IV) stages except for hK5 $(P=0.007)$; higher fractionally ranked hK5 predicts FIGO stage III/IV versus I/II with OR $7.75[95 \%$ confidence interval (95\% CI), 1.7-35.5]. Similarly, of the antigens, only hK5 was significantly associated $(P=0.004)$ with nuclear grade (higher hK5 implying a tendency to poorer grade).

Progression-free survival. Among the clinical variables entered into Cox models for PFS in all patients (Table 3), univariate predictors were RT, ascites volume, nodal status, FIGO III/IV versus I/II, and FIGO IV versus I/II/III. Among the proteolytic factors, hK11 had borderline significance, with high values being favorable ( $\mathrm{HR}, 0.44 ; 95 \% \mathrm{CI}, 0.19-1.02 ; P=$ 0.055 ). It was significant in $\mathrm{G} 2 / \mathrm{G} 3$ patients (HR, 0.40; 95\% CI,

Table 3. Univariate and multivariate Cox proportional hazard regression analyses for PFS in all patients $(N=142)$

Variable

Univariate

Multivariate

RT presence

FIGO III/IV vs. I/II

FIGO IV vs. I/II/III

Ascites volume $>500 \mathrm{~mL}$

Nodal status

Fractionally ranked PAI-1 - fractionally rank uPA

Fractionally ranked hK11

\begin{tabular}{lrrrr}
\multicolumn{2}{c}{ Univariate } & & \multicolumn{2}{c}{ Multivariate } \\
HR (95\% CI) & $\boldsymbol{P}$ & & HR (95\% CI) & $\boldsymbol{P}$ \\
\hline $4.37(2.55-7.47)$ & $<0.001$ & $4.53(2.59-7.95)$ & $<0.001$ \\
$9.25(2.25-38.1)$ & 0.002 & - & $\mathrm{NS}$ \\
$2.18(1.25-3.82)$ & 0.006 & - & $\mathrm{NS}$ \\
$2.37(1.45-3.88)$ & 0.001 & - & $\mathrm{NS}$ \\
$1.87(1.11-3.17)$ & 0.019 & - & $\mathrm{NS}$ \\
$1.98(1.01-3.89)$ & 0.046 & $0.30(0.11-0.76)$ & 0.011 \\
$0.44(0.19-1.02)$ & 0.055 & &
\end{tabular}

NOTE: Variables entered were FIGO (I-IV), RT presence, ascites volume, age, nuclear grade, nodal status, fractionally ranked antigen levels uPA, PAI-1, and the difference of their fractional ranks, hK5, hK6, hK7, hK8, hK10, hK11, and hK13. All were coded as described in Statistical Methods. Fractionally ranked hK11 was significant in the multivariate model, although only borderline significant in univariate analysis. Although FIGO variables are strong univariate survival factors, they are not significant in the multivariate model (see text).

Abbreviation: NS, not significant. 
Table 4. Univariate and multivariate Cox proportional hazard regression analyses for OS in all patients

Univariate

\begin{tabular}{c}
\hline HR (95\% CI) \\
$8.23(4.20-16.0)$ \\
$6.35(1.96-20.6)$ \\
$3.32(1.94-5.67)$ \\
$3.52(2.12-5.87)$ \\
$3.73(1.98-7.01)$ \\
$2.07(1.19-3.62)$ \\
$0.41(0.17-0.99)$ \\
$0.27(0.08-0.85)$
\end{tabular}

Multivariate

\begin{tabular}{cc}
\hline HR (95\% CI) & $\boldsymbol{P}$ \\
\hline $7.49(3.63-15.45)$ & $<0.001$ \\
- & NS \\
- & NS \\
$1.97(1.12-3.46)$ & 0.018 \\
- & NS \\
- & NS \\
$0.196(0.072-0.54)$ & 0.002 \\
- & NS
\end{tabular}

NOTE: Variables entered were FIGO (I-IV), RT presence, ascites volume, age, nuclear grade, nodal status, and fractionally ranked antigen levels uPA, PAI-1, hK5, 6, 7, 8, 10, 11 and 13, coded as described in Statistical Methods. HR for antigens refers to fractional ranks. Although FIGO variables are strong univariate survival factors, they are not significant in the multivariate model.

0.17-0.96; $P=0.039$, not shown). The fractional rank difference between PAI-1 and UPA had significant univariate effect (HR, $1.98 ; 95 \% \mathrm{CI}, 1.01-3.89 ; P=0.046)$ on PFS. This difference was also significant and a bit stronger in the subgroup of patients with RT (HR, 2.86; 95\% CI, 1.19-6.84; $P=0.018$, not shown) but not in the optimally debulked subgroup.

In multivariate analysis of PFS in all patients (Table 3), RT had HR >4.5; neither FIGO status nor the other clinical variables entered the model, whereas of the antigens hK11 alone was significant. To illustrate HR 0.30 for fractional ranks, consider two hypothetical patients with equal RT but hK11 levels of $6.2 \mathrm{ng} / \mathrm{mg}$ (75th percentile) and $0.1 \mathrm{ng} / \mathrm{mg}$ (25th percentile), respectively. Because these fractional ranks differ by 0.5 , their HR would be $(0.30)^{0.5} \approx 0.55$.

Overall survival. For OS in all patients (Table 4), univariate predictors were RT, FIGO stages nodal status, ascites volume, nuclear grade, as well as hK10 and hK13. The clinical factors, RT (HR, 7.49) and ascites volume (HR, 1.97), but not FIGO stage, as well as antigen hK10 (HR, 0.196) enter multivariate OS model.

$R T$ presence. Due to the strong effect of surgical success on PFS and OS, any factor influencing surgical success could affect survival, independent of its significance in multivariate PFS and OS models. Clinical factors ascites volume, age, nuclear grade, and nodal status, as well as fractionally ranked antigens, were entered into univariate and multivariate logistic regression for RT (Table 5). Ascites volume (OR, 8.92), nuclear grade (OR, 3.01), and hK5 (HR, 1.52) were statistically significant univariate factors. In multivariate models excluding clinical factors but including the proteolytic factors, higher hK5 (OR, 6.40) and lower hK13 (OR, 0.16) were associated with unfavorable surgical outcome. Entering the relevant clinical and proteolytic factors, one obtains the multivariate scoring model for RT on the right of Table 5. Of the clinical factors, larger ascites volume $(\mathrm{OR}, 13.13)$ and poorer nuclear grade $(\mathrm{OR}, 2.92)$ were associated with poorer surgical outcome; regarding the antigens, higher hK6 $(\mathrm{OR}, 8.54)$ and lower hK13 (OR, 0.14) contributed to poorer surgical outcome. The model provides risk assessment for RT based on ascites volume, nuclear grade, hK6, and hK13, which could be estimated before surgery. Figure 1 shows the ROC (sensitivity, specificity for RT) of the probability score computed from this logistic regression model; its area under ROC of 0.833 illustrates the in-sample modeling quality.

\section{Discussion}

Elevated serum or tissue levels of hKs have been individually implicated as diagnostic and prognostic factors in ovarian cancer (24). To enhance their usefulness for clinical decisions, this article has evaluated the multivariate effect of seven hKs (hK5, hK6, hK7, hK8, hK10, hK11, and hK13), uPA, and its inhibitor PAI-1, as well as clinical and histomorphologic variables on survival and surgical success, in a collective of 142 FIGO stage I to IV ovarian cancer patients.

In both univariate and multivariate analysis, RT was a decisive (unfavorable) clinical determinant of PFS and OS, in accordance with a meta-analysis (25).

In univariate analysis (PFS), aside from RT, ascites volume and nodal status were significant clinical variables; the fractional rank difference of PAI-1 and uPA (i.e., high PAI-1 compared with uPA) was significant for poor PFS; hK11 was of borderline significance; none of the other proteolytic factors was significant. In multivariate analysis (PFS), hK11 was the only statistically significant biomarker, taking RT into account. Elevated hK11 tumor antigen levels were associated with prolonged PFS.

In previous studies, the effect of hK11 in ovarian cancer depended on whether protein or mRNA levels were considered: KLK11 mRNA expression levels were associated with poor survival in ovarian cancer (26). However, in agreement with our findings, elevated tissue hK11 antigen levels determined by a similar ELISA were favorable for PFS (14).

Our group previously found that uPA and PAI-1 are significant prognostic factors regarding OS in FIGO IIIC ovarian cancer. Although uPA was univariately significant, its effect was not seen in multivariate analysis; the effect of PAI-1 was time varying and more pronounced within the first 2 years (2). In the present collective, high PAI-1 compared with uPA was significantly associated with poor PFS, particularly in the subgroup of patients with RT. These results taken together suggest that PAI-1 and uPA could play important but distinct roles in the complex process of tumor invasion. Additionally (aside from the 41 patients in common), the patients in the present collective were treated more recently, in particular applying a revised chemotherapy standard (carboplatin/paclitaxel as opposed to the earlier carboplatin/cyclophosphamide). If $\mathrm{uPA}$ and/or PAI-1 were associated with response to 
Table 5. Logistic regression analyses for presence of RT mass

\begin{tabular}{|c|c|c|c|c|c|c|}
\hline \multirow[t]{2}{*}{ Variable } & \multicolumn{2}{|c|}{ Univariate } & \multicolumn{2}{|c|}{ Multivariate (antigens only) } & \multicolumn{2}{|c|}{ Multivariate with clinical } \\
\hline & OR (95\% CI) & $\boldsymbol{P}$ & OR $(95 \% \mathrm{CI})$ & $\boldsymbol{P}$ & OR $(95 \% \mathrm{CI})$ & $\boldsymbol{P}$ \\
\hline Ascites volume $>500 \mathrm{~mL}$ & $8.92(3.67-21.7)$ & $<0.001$ & \multicolumn{2}{|c|}{ Not entered } & $13.13(4.50-38.2)$ & $<0.001$ \\
\hline Nuclear grade & $3.01(1.40-6.40)$ & 0.005 & \multicolumn{2}{|c|}{ Not entered } & $2.92(1.13-7.5)$ & 0.027 \\
\hline Fractionally ranked hK5 & $1.52(1.25-16.6)$ & 0.021 & $6.40(1.63-25.0)$ & 0.008 & - & NS \\
\hline Fractionally ranked hK6 & - & NS & - & NS & $8.54(1.57-46.4)$ & 0.013 \\
\hline Fractionally ranked hK13 & - & NS & $0.16(0.03-0.74)$ & 0.019 & $0.14(0.02-0.93)$ & 0.042 \\
\hline
\end{tabular}

NOTE: Factors entered in analyses "univariate" and "multivariate with clinical" were clinical variables ascites volume, age, and nuclear grade, as well as fractionally ranked antigens UPA, PAI-1, and the tissue kallikreins. Variables entered in analysis "multivariate (antigens only)" were fractionally ranked antigens UPA, PAI-1, and the tissue kallikreins. In univariate analysis, OR is unadjusted, whereas in multivariate analyses, OR is adjusted. ORs refer to the coding as described in Statistical Methods; in particular, OR of hK5, hK6, and hK13 refer to fractional ranks. All variables entering models could be estimated before surgery.

carboplatin/paclitaxel therapy, this "predictive" association could have modified their apparent "prognostic" significance. This hypothesis, although not previously discussed with respect to $\mathrm{UPA}$ and PAI-1 in ovarian cancer, is reminiscent of the situation in breast cancer (27).

In multivariate analysis, low hK10 levels, RT, and ascites volume were independently associated with poor OS. It is noteworthy that although high hK13 was a good univariate predictor of improved OS, it was not significant in the multivariate model upon inclusion of RT; hence, the univariate effect may be partly attributable to its ability to predict absence of RT mass. In contrast, elevated hK10 is an even stronger predictor of favorable OS in a multivariate model that includes RT than in univariate analysis of OS. These findings seem consistent with hK10 down-regulation during tumor progression in nude mice inoculated with breast or prostate cancer cell lines $(28,29)$.

Unfavorable outcome in FIGO III/IV ovarian cancer was reported to be associated with hK10 tissue levels (30) exceeding a cutoff; however, hK10 was neither significant as a continuous variable nor in multivariate analysis including all FIGO I-IV patients. In view of the still unresolved underlying biological complexity, a nonmonotonic relationship between hK10 and tumor aggressiveness cannot be ruled out. The partly discrepant results in the literature indicate that the existing data on the diagnostic and prognostic role of hKs in ovarian cancer merit further investigation. Differences in patient selection, treatment, determination methods, and use of optimal cutoffs could be relevant.

Because chemotherapy (platinum compounds \pm taxane) was rather homogeneously administered to our primary ovarian cancer patients, the observed effect of tumor biological factors on survival (PFS, OS) reflects a superposition of their biological role in the natural course of disease and their influence on therapy response: The observed survival effect of a factor could be modified or masked by a predictive component with respect to therapy response. However, because ovarian cancer spreading is primarily locoregional, factors for tumor aggressiveness are reflected not only in survival but also already in the tumor burden within the abdominal cavity. Hence, studying relationships between tumor-associated factors and probability of surgical success reveals key information on localized tumor aggressiveness free of confounding effects of therapy response. Moreover, because completeness of cytoreductive surgery is a key determinant of outcome, preoperative determination of factors influencing surgical success may be helpful in individualizing treatment of ovarian cancer, for example, selection of patients for second surgical approach after incomplete primary surgery or for administration of preoperative chemotherapy.

Despite recent improvements, ovarian cancer mortality remains high. FIGO reports 5-year survival of $28.9 \%$ for FIGO IIIC and $13.4 \%$ for FIGO IV (31).

Survival in advanced ovarian cancer is better for patients without macroscopic RT after primary surgery compared with patients with RT $(25,32,33)$. However, radical tumor resection may cause considerable morbidity (e.g., postoperative subileus, relaparotomy due to anastomosis defects, cardiorespiratory failure, thromboembolism, sepsis, or lymphoceles). Systematic lymph node dissection-a recommended procedure when optimal debulking seems achievable-by itself often causes significantly higher blood loss and consecutive transfusion rates compared with surgery without radical lymphadenectomy (34). Our overall morbidity rate after advanced ovarian cancer

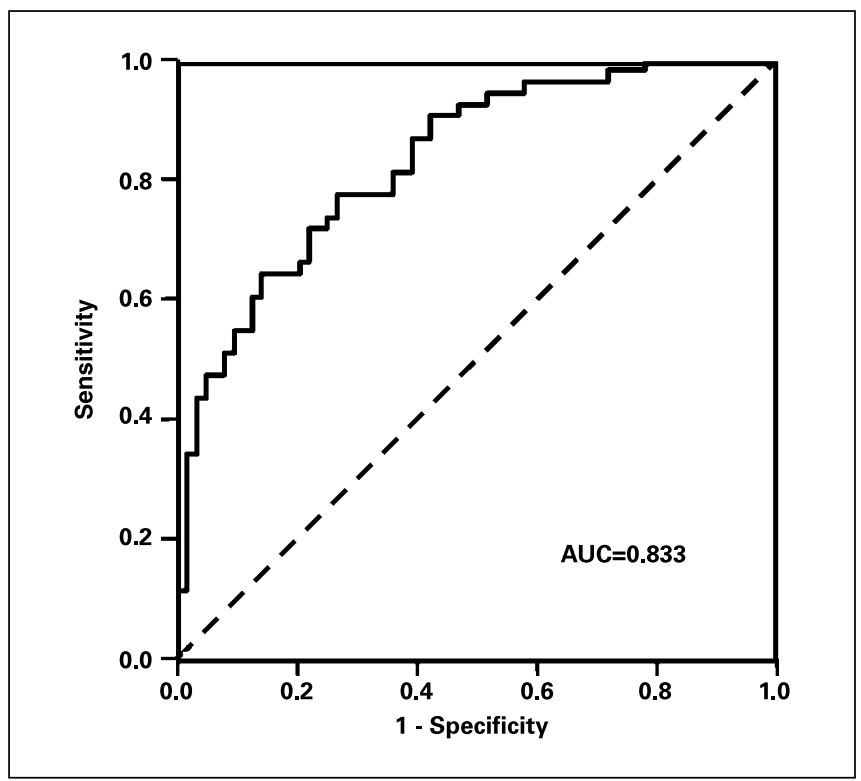

Fig. 1. ROC for prediction of RT, based on the model of Table 5 and including ascites volume, nuclear grade, and tissue kallikreins hK6 and hK13. Area under the ROC curve is 0.833 . Note that this is an in-sample estimate. 
surgery is $\sim 40 \%$, but radical surgery may lead to up to $80 \%$ morbidity (35). High morbidity is certainly acceptable if optimal debulking with its attendant survival advantage can be achieved; however, it must be viewed critically for patients in whom optimal debulking was not achievable and who thus have a short remaining life expectancy.

To estimate risks and benefits of surgical interventions preoperatively, clinical or biological factors are urgently needed that could more accurately predict individual debulking success. In ovarian cancer, tumor biological processes may strongly affect the degree of diffusion and dissemination of tumor cells within the abdominal cavity and hence influence the chance for surgical success. Indeed, in our study, besides large ascites volume and high nuclear grade, high hK5 univariately predicted poor surgical success. Moreover, in multivariate analysis, besides favorable grade and low ascites volume, low hK6 and high hK13 predicted surgical success. We previously described the predictive effect of preoperative ascites for surgical outcome in FIGO IIIc ovarian cancer (2). Regarding hKs, there is limited information regarding their effect on surgical success: For elevated hK10, a significant association with large RT was reported (30); for hK11, a significant correlation with response to chemotherapy, but not with surgical outcome was observed (14).

Using a logistic regression model, we have now obtained a score (RT score) based on hK6, hK13, ascites volume, and nuclear grade that predicted surgical outcome with good performance (area under ROC, 0.833). Such a score could be calculated before definitive surgery: preoperative ascitic fluid volume is easily estimated by ultrasound; tumor biopsies can be obtained for tissue analysis. The score could support preoperative risk stratification: In patients with considerable comorbidity, a favorable RT score would reinforce the decision for radical surgery, whereas an unfavorable RT score might indicate an alternative therapeutic approach, such as preoperative chemotherapy. Up to now, preoperative chemotherapy is not a standard of care in the management of ovarian cancer. But recruitment of the European Organization for Research and Treatment of Cancer protocol 55971 comparing upfront debulking surgery versus neoadjuvant chemotherapy in patients with stage IIIc or IV epithelial ovarian cancer in a randomized phase III trial is almost completed. This trial will elucidate whether neoadjuvant chemotherapy is an alternative approach with the same outcome compared with the standard procedure, upfront surgery $(36,37)$. Thus far, no reliable parameters exist to predict which patients are likely to benefit from preoperative chemotherapy. In a pilot study, we showed that patients with large ascites volume who received preoperative chemotherapy followed by radical surgery had less RT at time of definitive surgery and a substantial survival advantage over comparable patients receiving chemotherapy after surgery (38). A predictive score combining tumor biological with clinical information-in our case using tissue kallikreins hK6, hK13, ascites volume, and nuclear grade-may thus help to identify suitable candidates for preoperative chemotherapy. It would be useful to test the validity of the predictive score by prospective measurement of these factors in a study using current treatment guidelines. With a validated scoring model for surgical success in ovarian cancer, the next step would then be to conduct a clinical trial designed to test whether patients benefit from treatment strategies that incorporate the new scoring information.

In conclusion, our study shows that some of the hKs are significant independent prognostic and predictive markers in ovarian cancer. Their role in disease progression is evidently complex, as different tissue kallikreins have a strong effect for different disease end points. Nevertheless, they have the potential to support clinical therapy decisions.

\section{References}

1. van der Burg $M$, Henzen-Logmans SC, Berns $E$, van Putten WL, Klijn JG, Foekens JA. Expression of urokinase-type plasminogen activator (UPA) and its inhibitor PAI-1 in benign, borderline, malignant primary and metastatic ovarian tumors. Int J Cancer 1996; 69:475-9

2. Kuhn W, Schmalfeldt B, Reuning U, et al. Prognostic significance of urokinase (UPA) and its inhibitor PAI-1 for survival in advanced ovarian carcinoma stage FIGO Illc. Br J Cancer 1999;79:1746-51.

3. Schmalfeldt B, Prechtel D, Harting K, et al. Increased expression of matrix metalloproteinases (MMP) -2, MMP-9, and the urokinase-type plasminogen activator is associated with progression from benign to advanced ovarian cancer. Clin Cancer Res 2001; 7: $2396-404$.

4. Konecny G, Untch M, Pihan A, et al. Association of urokinase-type plasminogen activator and its inhibitor with disease progression and prognosis in ovarian cancer. Clin Cancer Res 2001;7:1743-9.

5. Borgono CA, Michael IP, Diamandis EP. Human tissue kallikreins: physiologic roles and applications in cancer. Mol Cancer Res 2004;2:257-80.

6. Yousef GM, Polymeris ME, Yacoub GM, et al. Parallel overexpression of seven kallikrein genes in ovarian cancer. Cancer Res 2003;63:2223-7.

7. Rosen DG, Wang L, Atkinson JN, et al. Potential markers that complement expression of CA125 in epithelial ovarian cancer. Gynecol Oncol 2005;99: 267-77.

8. Diamandis EP, Scorilas A, Fracchioli S, et al. Human kallikrein 6 (hK6): a new potential serum biomarker for diagnosis and prognosis of ovarian carcinoma. J Clin Oncol 2003;21:1035-43.

9. Luo LY, Katsaros D, Scorilas A, et al. The serum concentration of human kallikrein 10 represents a novel biomarker for ovarian cancer diagnosis and prognosis. Cancer Res 2003:63:807-11.

10. Diamandis EP, Borgono CA, Scorilas A, et al. Immunofluorometric quantification of human kallikrein 5 expression in ovarian cancer cytosols and its association with unfavorable patient prognosis. Tumour Biol 2003;24:299-309.

11. Kishi T, Grass L, Soosaipillai $A$, et al. Human kallikrein 8 , a novel biomarker for ovarian carcinoma. Cancer Res 2003;63:2771 -4.

12. Diamandis EP, Borgono CA, Scorilas A, Harbeck N Dorn J, Schmitt M. Human kallikrein 11: an indicator of favorable prognosis in ovarian cancer patients. Clin Biochem 2004;37:823-9.

13. Scorilas A, Borgono CA, Harbeck N, et al. Human kallikrein 13 protein in ovarian cancer cytosols: a new favorable prognostic marker. J Clin Oncol 2004;22: $678-85$

14. Borgono CA, Fracchioli S, Yousef GM, et al. Favorable prognostic value of tissue human kallikrein 11 (hK11) in patients with ovarian carcinoma. Int J Cancer 2003;106:605-10.

15. Schmitt M, Sturmheit AS, Welk A, Schnelldorfer C, Harbeck N. Procedures for the quantitative protein determination of urokinase and its inhibitor, PAI-1, in human breast cancer tissue extracts by ELISA. Methods Mol Med 2006;120:245-65.

16. Kuhn W, Pache L, Schmalfeldt B, et al. Urokinase
(UPA) and PAl-1 predict survival in advanced ovarian cancer patients (FIGO III) after radical surgery and platinum-based chemotherapy. Gynecol Oncol 1994; 55:401-9.

17. Christopoulos TK, Diamandis EP. Enzymatically amplified time-resolved fluorescence immunoassay with terbium chelates. Anal Chem 1992;64:342-6.

18. Yousef GM, Polymeris ME, Grass L, et al. Human kallikrein 5: a potential novel serum biomarker for breast and ovarian cancer. Cancer Res 2003;63: 3958-65.

19. Diamandis EP, Yousef GM, Soosaipillai AR, Bunting P. Human kallikrein 6 (zyme/protease $\mathrm{M} /$ neurosin): a new serum biomarker of ovarian carcinoma. Clin Biochem 2000:33:579-83.

20. Kishi T, Soosaipillai A, Grass L, Little SP, Johnstone EM, Diamandis EP. Development of an immunofluorometric assay and quantification of human kallikrein 7 in tissue extracts and biological fluids. Clin Chem 2004;50:709-16.

21. Kishi T, Grass L, Soosaipillai A, Shimizu-Okabe C, Diamandis EP. Human kallikrein 8: immunoassay development and identification in tissue extracts and biological fluids. Clin Chem 2003;49:87-96.

22. Luo LY, Grass L, Howarth DJ, Thibault $P$, Ong $H$, Diamandis EP. Immunofluorometric assay of human kallikrein 10 and its identification in biological fluids and tissues. Clin Chem 2001;47:237-46.

23. Kapadia C, Chang A, Sotiropoulou G, et al. Human kallikrein 13: production and purification of recombinant protein and monoclonal and polyclonal antibodies, and development of a sensitive and 
specific immunofluorometric assay. Clin Chem 2003; 49:77-86.

24. Borgono CA, Diamandis EP. The emerging roles of human tissue kallikreins in cancer. Nat Rev Cance 2004:4:876-90.

25. Bristow RE, Tomacruz RS, Armstrong DK, Trimble EL, Montz FJ. Survival effect of maximal cytoreductive surgery for advanced ovarian carcinoma during the platinum era: a meta-analysis. J Clin Oncol 2002 $20: 1248-59$

26. Shigemasa K, Gu L, Tanimoto $H, O^{\prime}$ Brien TJ, Ohama K. Human kallikrein gene 11 (KLK11) mRNA overexpression is associated with poor prognosis in patients with epithelial ovarian cancer. Clin Cancer Res 2004 10:2766-70.

27. Harbeck N, Kates R, Look M, et al. Enhanced benefit from adjuvant chemotherapy in breast cance patients classified high-risk according to urokinasetype plasminogen activator (UPA) and plasminogen activator inhibitor type $1(n=3424)$. Cancer Res 2002;62:4617-22.

28. Liu XL, Wazer DE, Watanabe K, Band V. Identification of a novel serine protease-like gene, the expres- sion of which is down-regulated during breast cancer progression. Cancer Res 1996;56:3371 -9.

29. Goyal J, Smith KM, Cowan JM, Wazer DE, Lee SW, Band V. The role for NES1 serine protease as a novel tumor suppressor. Cancer Res 1998:58: 4782-6

30. Luo LY, Katsaros D, Scorilas A, et al. Prognostic value of human kallikrein 10 expression in epithelial ovarian carcinoma. Clin Cancer Res 2001;7: 2372-9.

31. Heintz APM, Odicino F, Maisonneuve $P$, et al. Carcinoma of the ovary. In: FIGO annual report on the results of treatment in gynecological cancer. Int $\mathrm{J}$ Gynaecol Obstet 2002;83 Suppl 1:135-66.

32. du Bois A, Luck HJ, Meier W, et al. A randomized clinical trial of cisplatin/paclitaxel versus carboplatin/ paclitaxel as first-line treatment of ovarian cancer. J Natl Cancer Inst 2003;95:1320-9.

33. Hoskins WJ, McGuire WP, Brady MF, et al. The effect of diameter of largest residual disease on survival after primary cytoreductive surgery in patients with suboptimal residual epithelial ovarian carcinoma. Am J Obstet Gynecol 1994;170:974-9.
34. Benedetti-Panici $P$, Maggioni A, Hacker N, et al. Systematic aortic and pelvic lymphadenectomy versus resection of bulky nodes only in optimally debulked advanced ovarian cancer: a randomized clinical trial. J Natl Cancer Inst 2005;97:560-6.

35. Eisenkop SM, Friedman RL, Wang HJ. Complete cytoreductive surgery is feasible and maximizes survival in patients with advanced epithelial ovarian cancer: a prospective study. Gynecol Oncol 1998;69: $103-8$.

36. Vergote I, van GorpT, Amant $F$, Neven $P$, Berteloot $P$. Neoadjuvant chemotherapy for ovarian cancer. Oncology (Huntingt) 2005;19:1615-22.

37. van Gorp $T$, Amant $F$, Neven $P$, Berteloot $P$, Leunen $\mathrm{K}$, Vergote I. The position of neoadjuvant chemotherapy within the treatment of ovarian cancer. Minerva Gynecol 2006;58:393-403

38. Kuhn W, Rutke S, Spathe K, Schmalfeldt B, et al. Neoadjuvant chemotherapy followed by tumor debulking prolongs survival for patients with poor prognosis in International Federation of Gynecology and Obstetrics Stage IIIC ovarian carcinoma. Cancer 2001;92:2585-91. 